\title{
A Solar Aspect System for the HEROES Mission
}

\author{
Steven Christe, Albert Shih, \\ Marcello Rodriguez, Kyle Gregory, \\ Alexander Cramer, Melissa Edgerton NASA Goddard Space Flight Center Greenbelt, MD 20771, USA \\ Telephone: (301) 286-7999 \\ Email: steven.christe@nasa.gov \\ Jessica Gaskin, Brian O'Connor, Alexander Sobey NASA Marshall Space Flight Center \\ Huntsville, AL 35811, USA \\ Telephone: (256) 961-7818 \\ Email: jessica.gaskin@nasa.gov
}

\begin{abstract}
A new Solar Aspect System (SAS) has been developed to provide the ability to observe the Sun on an existing balloon payload HERO (short for High Energy Replicated Optics). Developed under the HEROES program (High Energy Replicated Optics to Explore the Sun), the SAS aspect system provides solar pointing knowledge in pitch, yaw, and roll. The required precision of these measurements must be better than the HEROES X-ray resolution of $\sim 20$ arcsec Full Width at Half Maximum (FWHM) so as to not degrade the image resolution. The SAS consists of two separate systems: the Pitch-Yaw Aspect System (PYAS) and the Roll Aspect System (RAS). The PYAS functions by projecting an image of the Sun onto a screen with precision fiducials. A CCD camera takes an image of these fiducials, and an automated algorithm determines the location of the Sun as well as the location of the fiducials. The spacing between fiducials is unique and allows each to be identified so that the location of the Sun on the screen can be precisely determined. The RAS functions by imaging the Earth's horizon in opposite directions using a silvered prism imaged by a CCD camera. The design and first results of the performance of these systems during the HEROES flight which occurred in September 2013 are presented here.
\end{abstract}

Index Terms - aspect system, high-altitude balloon, solar, Sun, X-ray

\section{CONTEnTS}

\section{Introduction}

II The HEROES Payload

III Pointing Requirements

IV Flight Overview

V The Solar Aspect System (SAS)

VI The Pitch-Yaw Aspect System (PYAS) 3

VI-A PYAS to Telescope Alignment . . . . . 3

VI-B The PYAS Algorithm ........ 3

VI-C Flight Performance .......... 5

U.S. Government work not protected by U.S. copyright
VII The Roll Aspect System (RAS) 6

VII-A Flight Performance . . . . . . . . . 7

VII-B Comparison to the Star Camera . . . 7

$\begin{array}{ll}\text { VIII Conclusion } & 7\end{array}$

References

Biographies 9

Steven Christe .............. 9

Albert Y. Shih . . . . . . . . . . . . 9

Marcello Rodriguez . . . . . . . . . . . . . 9

Kyle Gregory . . . . . . . . . . . . . . . . . . . . . . . . . . . . 9

Alexander Cramer . . . . . . . . . . . . 9

Melissa Edgerton . . . . . . . . . . . 9

Jessica Gaskin . . . . . . . . . . . . . 9

Brian O'Connor . . . . . . . . . . . 9

Alexander Sobey . . . . . . . . . . . 9

\section{INTRODUCTION}

The High Energy Replicated Optics to Explore the Sun (HEROES) mission is a collaboration between NASA's Marshall Space Flight Center (MSFC) and the Goddard Space Flight Center (GSFC) to upgrade and fly an existing MSFCdeveloped balloon payload to make novel hard x-ray (HXR) solar observations during the day and astrophysical observations at night during a single flight. HEROES builds upon the existing MSFC-developed HERO payload by adding the ability to observe the Sun through the addition of a new Solar Aspect System (SAS) and a number of other improvements [1], [2]. HERO has flown several times in the past, most recently in 2011 from Alice Springs, Australia, to observe the Crab Nebula under the leadership of Dr. B. Ramsey [3]. The HEROES project is funded by the NASA HOPE (Hands On Project Experience) Training Opportunity awarded by the NASA Academy of Program/Project and Engineering Leadership, in partnership with NASA's Science Mission Directorate, Office of the Chief Engineer and Office of the Chief Technologist. 


\section{THE HEROES PAYLOAD}

The HEROES payload is an upgrade of the existing HERO payload. The payload is comprised of a gondola and support systems as well as the HEROES telescope, which consists of 8 grazing-incidence HXR telescopes with a 6-m focal length coupled to complementary gas scintillation proportional counter detectors, all held by a carbon-fiber and aluminum optical bench. A diagram of the payload can be seen in Figure 1.

The gondola makes use of a coarse aspect system for slewing based on rate-controlled gyros. An elevation (i.e., pitch) motor controls the elevation by tipping the optical bench about its center. An azimuth (i.e., yaw) motor is located at the top of the gondola structure and rotates the entire payload. A differential global positioning system (GPS) and a magnetometer provide feedback for pointing in azimuth. A fine inertial-mode pointing system has historically been based upon aspect information provided by a day/night co-aligned star camera to observe astrophysical sources. These aspect solutions are used to update the gyro drift rates to keep the target in the center of the telescope's field of view. Due to the extreme sensitivity necessary to observe stars, the HEROES star camera does not have the ability to provide aspect while pointing near ( $<1$ degree) the Sun.

Two payload upgrades provide the capability to observe the Sun and astrophysical targets during the same balloon flight. First, the addition of a new Solar Aspect System (SAS) provides real-time solar aspect feedback to the fine inertialmode pointing as well as high-precision post-flight pointing knowledge. Second, a removable shutter was added to the star camera to protect it during solar observations.

\section{Pointing REQUiREMEnTS}

The nature of the HEROES HXR optics combined with the existing HERO pointing control system dictated the HEROES solar pointing requirements. The HEROES HXR telescope is an imaging telescope which operates in photon-counting mode. The arrival time, location, and energy of each photon is recorded individually. Therefore, the stability of the platform is not constrained by an exposure time. On the other hand, relative-pointing knowledge must be accurate in order to reconstruct images post-flight. The resolution of the telescopes is $\sim 20$ arcsec. Pitch-yaw-aspect knowledge must therefore be commensurate with this value in order to not degrade the image resolution. A roll-knowledge requirement of 3.6 arcmin limits the effective additional pitch-yaw uncertainty to $<1$ arcsecond over the entire solar disk. A pitch-yawjitter requirement of within 1 arcmin of the target for $50 \%$ of the time is a consequence of the fact that the telescope's response degrades rapidly in both angular resolution as well as throughput as a function of off-axis angle. The real-time aspect solutions are required to be provided to the pointing control system with an update cadence of 1 second. Finally, the HEROES coarse aspect system can point HEROES to within $\sim 1$ degree of a target, which translates to a field-ofview requirement for the SAS of 2 degree diameter. See Table I for a summary of these requirements.

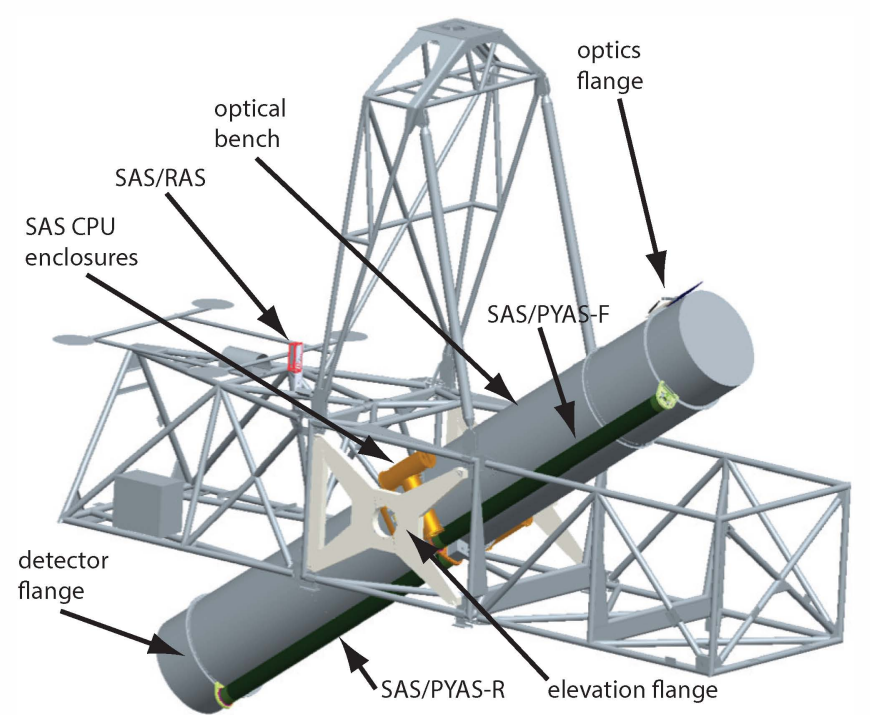

Fig. 1: A diagram showing the HEROES gondola along with the Solar Aspect System components, the Pitch-Yaw Aspect System (PYAS) and the Roll Aspect System (RAS). The PYAS is mounted to the side of the gondola co-boresighted with the main telescope. It is composed of two subsystems: the PYASF (front) and PYAS-R (rear). The RAS is a separate system mounted on a truss near the center of the gondola.

TABLE I: Summary of the HEROES SAS pointing requirements versus that achieved during flight

\begin{tabular}{lll}
\hline Type & Requirement & Achieved \\
\hline Pitch-Yaw Knowledge (Relative) & $20 \operatorname{arcsec}$ & $<10 \operatorname{arcsec}$ \\
Roll Knowledge (Relative) & $3.6 \operatorname{arcmin}$ & $<0.3 \operatorname{arcmin}$ \\
Pitch-Yaw Jitter (50\% of time) & 1 arcmin & $25 \operatorname{arcsec}$ \\
Co-alignment & $1 \mathrm{arcmin}$ & $\sim 1 \mathrm{arcmin}$ \\
Cadence & $1 \mathrm{~Hz}$ & $4 \mathrm{~Hz}$ \\
Field of View (Diameter) & 2 degrees & 2.91 degrees \\
\hline
\end{tabular}

\section{Flight OVERVIEW}

The HEROES payload was launched from the Columbia Scientific Ballooning Facility in Fort Sumner, NM, on 2013 Sep 21 (Flight \#645N) and spent approximately 21 hours at a float altitude greater than $32 \mathrm{~km}$. The launch took place at 05:58 local time (UTC-7). The payload officially reached float altitude $(129 \mathrm{kft})$ at 08:49. At 08:55, the payload began to slew to the Sun using the coarse pointing system. At 09:21, the PYAS-F began providing aspect solutions and the payload entered fine-pointing mode. Soon thereafter the SAS was commanded to point to a solar target at near the limb of the Sun. At 16:34, after 7 hours of solar observations, HEROES switched to making astrophysical observations.

\section{The Solar Aspect System (SAS)}

In order to meet the requirements listed in Table I, the SAS consists of two separate systems: the Pitch-Yaw Aspect System (PYAS) and the Roll Aspect System (RAS). The design concept is based on a similar system that is currently being developed for the GRIPS (Gamma-Ray Imaging/Polarimeter for Solar flares) balloon payload [4], which is scheduled to fly 
in 2014. The PYAS is composed of two subsystems, the PYASF (front) and the PYAS-R (rear). Either of these two nearly identical subsystems can provide pitch-yaw aspect knowledge as well as real-time pointing solutions to the fine inertial-mode pointing system. The RAS records data during flight for postflight determination of the roll aspect knowledge.

Two single-board computer stacks (called SAS1 and SAS2), each in its own air-tight enclosures pressurized to greater than 1 atm, controlled the SAS systems. SAS1 controlled PYAS-F, and SAS2 controlled both PYAS-R and RAS. Both SAS 1 and SAS2 computers used the Cool RoadRunner-945GSE PC/104Plus single-board computer, and each had two 250-GB solidstate drives to store images for post-flight processing. All three systems recorded their aspect images using the Imperx Bobcat IGV-B1310, a 1 megapixel Charge-Coupled Device (CCD) camera, controlled through an Ethernet connection. To maintain all systems within their operating temperatures, a passive thermal control architecture was used for the system, which consisted of optical coatings, heaters, and thermostats. More information about this aspect of the system is available [5].

\section{The Pitch-YaW Aspect System (PYAS)}

PYAS-F and PYAS-R together span the entire length of the 6-meter optical bench. In addition to providing redundancy, the two systems when compared provide a measurement of the misalignment between the x-ray optics and detectors during solar observations. The basic design of either PYAS subsystem (both front and rear) is as follows. A singlet plano-convex lens with a 3-m focal length (by OptoSigma) is located at the front of the subsystem and produces an image of the Sun onto a metal screen $3 \mathrm{~m}$ away. An IR (700 nm cutoff) filter plus a bandpass filter $(630 \pm 2 \mathrm{~nm}$ center, $10 \pm 2 \mathrm{~nm}$ Full Width at Half Maximum FWHM) were mounted in front of the solar lens. These filters reduce the solar heat input onto the screen and minimize chromatic aberration. A CCD camera with a 75$\mathrm{mm}$ lens set to $\mathrm{f} / 8$, located next to the solar lens, recorded the image of the Sun on the screen as a function of time with a cadence of $\sim 4 \mathrm{~Hz}$. A painted cardboard baffle spans the $3 \mathrm{~m}$ between the solar lens and the screen to block any scattered light. For PYAS-F (PYAS-R), the lens and camera are mounted at the optics (elevation) flange, and the screen is mounted at the elevation (detector) flange.

To be able to precisely determine the location of the image of the Sun on the screen using the camera, the screen was painted white and a deliberate pattern of black cross-shaped fiducials was added by screen printing (Figure 2). The use of fiducials on the screen greatly reduces the requirements on camera alignment. The screen plate scale is $1.1 \mathrm{arcmin} / \mathrm{mm}$ and spans 2.91 degrees. For reference, the Sun's angular size is $\sim 0.5$ degrees. With the PYAS camera system imaging the screen, each pixel corresponds to $\sim 10.2$ arcsec while the whole Sun is about 190 pixels across. Each image was processed in real-time to determine aspect solutions at a rate of $4 \mathrm{~Hz}$. Solutions were provided to the pointing control system at a rate of $1 \mathrm{~Hz}$. Every PYAS image was also saved to the solid-state storage for post-flight processing.

\section{A. PYAS to Telescope Alignment}

A requirement of any co-boresight pointing system is to ensure that both systems are pointing in the same direction. For the PYAS no adjustments can be made to the system since the solar lens and screen are hard mounted to the optical bench. The problem is then to find the calibrated PYAS screen center. A ground calibration procedure similar to that used for the HERO HXR telescopes and the star camera has been developed for the SAS. It makes use of the existing HERO alignment stand. This stand is used to precisely position light sources (HXR or visible) to be imaged by HEROES. To calibrate the screen center for PYAS-F and PYAS-R, a laser illuminated both solar lenses at the same time as HXRs were illuminating the HXR optics from 100 feet. Mechanical features on the alignment stand are accurate to $<1$ arcsec at this distance. The stand was leveled to better than 0.1 degrees, and initial alignment was made by using a set of two alignment holes on the side of the optical bench. The image of the laser spot on the PYAS screens defines the screen centers at the same time as the HXR telescope centers were measured. This method was found to provide the location of the screen center to within an accuracy of $\sim 1$ arcmin. This accuracy was limited by low-level distortions of the image introduced by the PYAS bandpass filter.

\section{B. The PYAS Algorithm}

The role of the PYAS algorithm is to process each image taken by the PYAS camera and produce a real-time aspect solution during solar observations. The image of the Sun, as well as the location of illuminated cross-shaped fiducials, are determined in pixel coordinates from the camera image. The physical locations of these fiducials are known from pre-flight measurements, and thus provide the translation of the Sun position to physical coordinates.

At a top level, the algorithm follows these steps:

1) Determine the location of the Sun in pixel coordinates

2) Determine the location of the Sun-illuminated fiducials in pixel coordinates

3) Identify visible fiducials in order to determine locations in payload coordinates

4) Generate the mapping from pixel coordinate to payload coordinates

5) Convert Sun location to payload coordinates

6) Convert payload coordinates to sky coordinates

Locating the Sun in a PYAS image is a shape-finding problem. The combination of image size, cadence, and limits on processing power ruled out traditional shape-finding methods like the Hough transform or template matching. In order to avoid operations on the entire PYAS image which are timeconsuming, the Sun-finding algorithm was based on the solaraspect system used by the RHESSI aspect system[6]. For each image frame, the algorithm examines a selection of 10-20 rows and columns evenly sampled across the entire image. In each of these lines, the algorithm searches for sharp increases followed later by a sharp decrease in brightness that indicates the presence of two solar-limb crossings. The solar limb is an extremely sharp feature $(<1$ arcsecond)[6]. As observed 


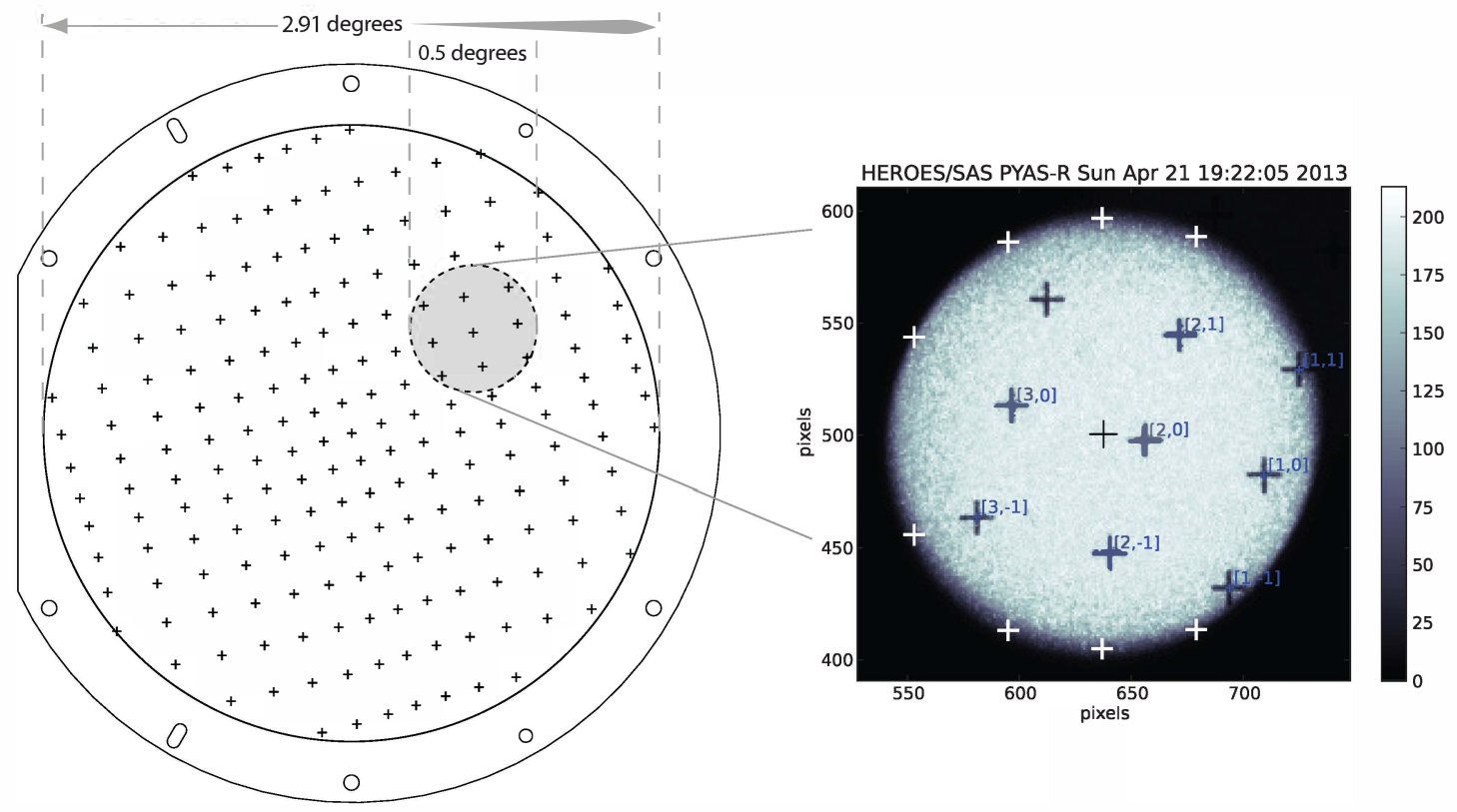

Fig. 2: (Left) The PYAS screen fiducial pattern. The active screen area is outlined in a circle. The outline represents the screen mounting area. The screen subtends 2.91 degrees of arc. The grey circle represents the approximate size of the Sun ( 0.5 degrees) on the screen. Since the fiducials are only visible on the small area of the screen illuminated by the Sun, the fiducial pattern was devised such that each fiducial can be uniquely identified by the distance to just two of its neighbors in perpendicular directions. (Right) An example image of the Sun as imaged onto the PYAS screen and recorded by the PYAS camera. Due to the limited dynamic range of the camera, the entire field of view of the camera is essentially black except for the Sun image. The sub image shows the Sun illuminating the fiducials on the PYAS screen. The white crosses show a few of the limb solutions found by the limb-finding algorithm. The thin black cross is the Sun center as derived from the set of limbs. The blue numbers show the results of the fiducial-finding algorithm, which has correctly identified all the fiducials and outputs their ID numbers.

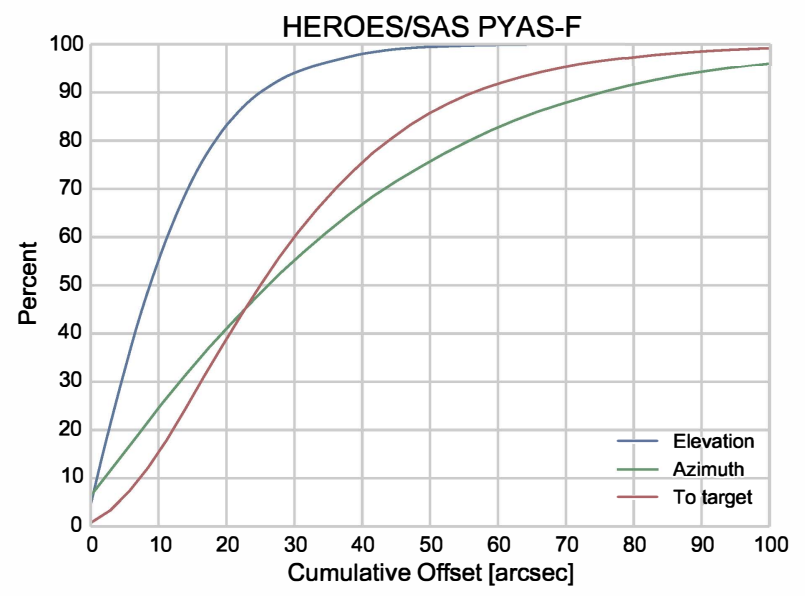

Fig. 4: The normalized cumulative distribution of offsets for the PYAS-F pointing in the elevation, azimuth, and distance to target in arcseconds. The pointing control in elevation and azimuth was found to be within 10 and 27 arcsec of the target, respectively. The overall pointing performance was found to be within $\sim 25 \operatorname{arcsec}$ of the target $50 \%$ of the time. by the PYAS camera, the limbs span approximately 8 pixels. The limb locations are refined with a linear fit to the intensity versus position, then averaged with each other to determine the Sun center. The standard deviation of the limb locations is used as the error for the location of the Sun center

Once the Sun has been located, the algorithm works on a sub-image containing just the region of the fiducial grid illuminated by the Sun (see Figure 2). Working with this smaller image allows the use of more computationally-intensive shapedetection algorithms [7]. The sub-image is convolved with a filter matched to the cross shape of a fiducial. The peaks of this response are refined by taking a thresholded centroid of the response around each peak value to determine sub-pixel locations of fiducials.

Once fiducials are located, they must be identified. The fiducials are arranged in a grid with a slight spiral (see Figure 2). The spacing between each row of fiducials increases monotonically from the center, and the spacing between each column of fiducials also increases monotonically from the center. With this fiducial strategy, the spacing between two fiducials in a line (either a row or a column) uniquely identifies those fiducials in that line. Thus, a given fiducial can be uniquely identified with just two neighboring fiducials in perpendicular directions. Once at least one fiducial has been uniquely identified, neighboring fiducials can be identified, 

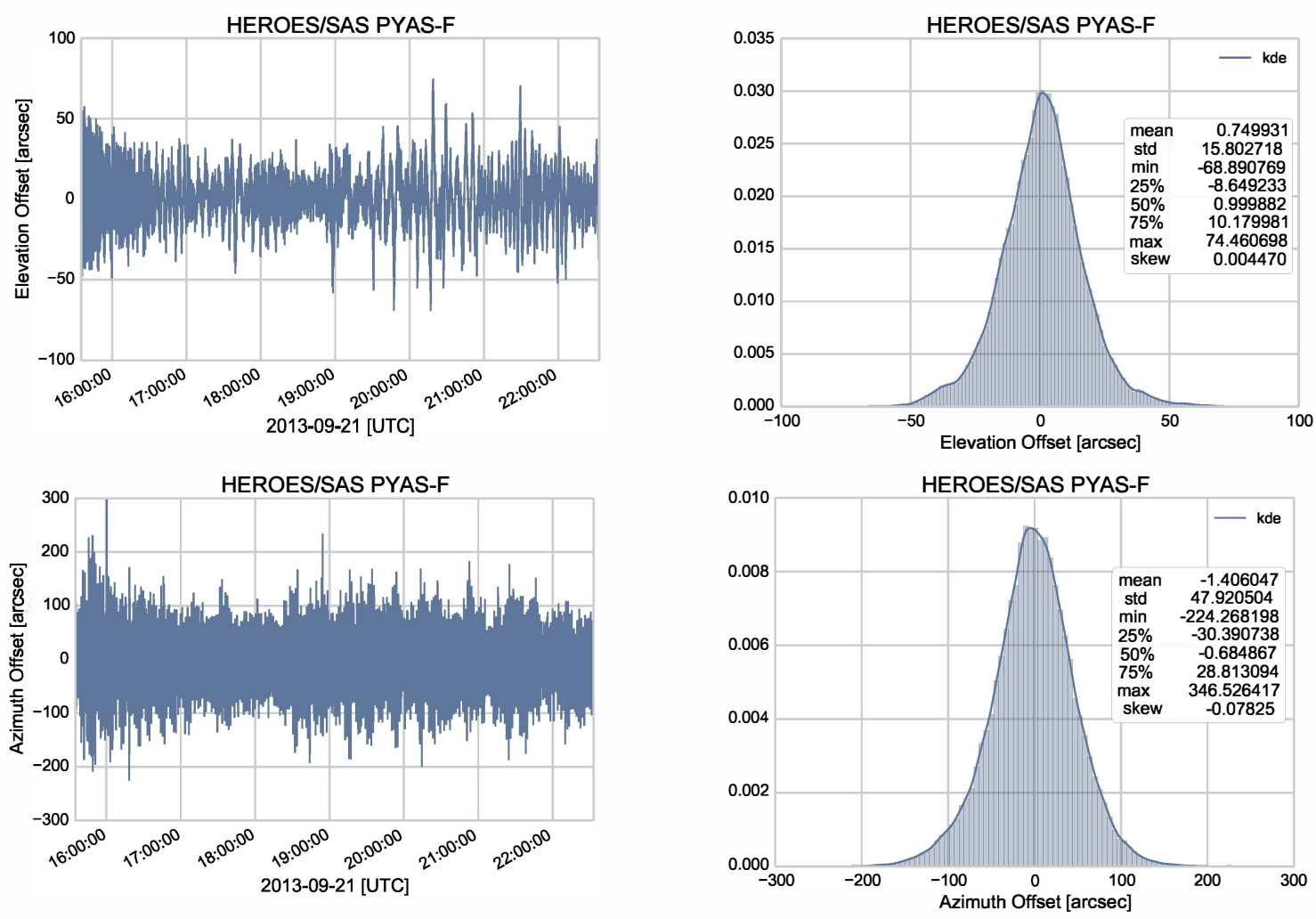

Fig. 3: (Top Left) A time series of the HEROES telescope target offset in elevation as measured by PYAS-F during the entirety of the HEROES solar observations. (Top Right) A histogram of the measured HEROES elevation by PYAS-F, which has a standard deviation of 16 arcsec. A kernel density estimator with a Gaussian kernel is overlaid for comparison. (Bottom Left) A time series of the HEROES telescope target offset in azimuth as measured by PYAS-F during the entirety of the HEROES solar observations. (Bottom Right) A histogram of the measured HEROES azimuth offset by PYAS-F, which has a standard deviation of 48 arcsec. A kernel density estimator with a Gaussian kernel is overlaid for comparison.

even if they do not have the necessary neighbors themselves.

Once fiducials are identified, their pixel to payload correspondences are used to compute a linear mapping between coordinate systems, and the resulting mapping is used to convert the pixel location of the Sun to payload coordinates. The implementation of this algorithm is open-source and can be found on https://github.com/HEROES-GSFC/SAS.

\section{Flight Performance}

As previously mentioned, HEROES solar observations lasted a total of 7 consecutive hours. During this time, only the PYAS-F system provided aspect solutions for solar pointing. The SAS1 computer saved a total of 103,820 images at an average rate of 3.98 images per second. Every fourth image was used to provide a real-time solution to the fine-pointing control system, for an update rate of $\sim 1 \mathrm{~Hz}$. During this time, PYAS-R was fully functional, but the PYAS-R solutions were not sent to the pointing control system. It achieved a slightly lower cadence of 3.9 images per second due to the fact that it also handles RAS images. Based on expected solar activity, the PYAS-F was commanded to target an active region near the limb of the Sun $([-794,102]$ arcsec from Sun center) for the entire duration of the solar observations. The pointing performance in elevation and azimuth can be seen in Figure 3 as well as Figure 4.

The time series of the elevation and azimuth target offsets for the entire solar pointing period is shown Figure 3 on the left. The time series presented here have been sub-sampled so as to manage the size of the file but only so far as it did not differ from the full plot as presented at this resolution. The pointing was found to be precise with few large excursions. Histograms of the pointing performance are shown on the right. A Gaussian kernel density estimator is overlaid ([8], [9]) for comparison and fits the data well. The means of the distribution in both azimuth and elevation were found to be small (-1.4 arcsec and 0.7 arcsec, respectively). The skews were also small (sub-arcsecond) which means that the distributions are symmetric about their mean showing that the payload was well balanced. The standard deviation of the pointing jitter from the target was found to be 16 arcsec and 48 arcsec in elevation and azimuth, respectively. The jitter in azimuth is significantly worse due to the fact that pointing in azimuth requires the entire gondola to move while pointing in elevation only requires tilting the optical bench.

The cumulative pointing distributions can be seen in Figure 4. The pointing control in elevation and azimuth was found to be within 10 and 27 arcsec of the target, respectively. The PYAS-F-controlled HEROES pointing was found to be within 


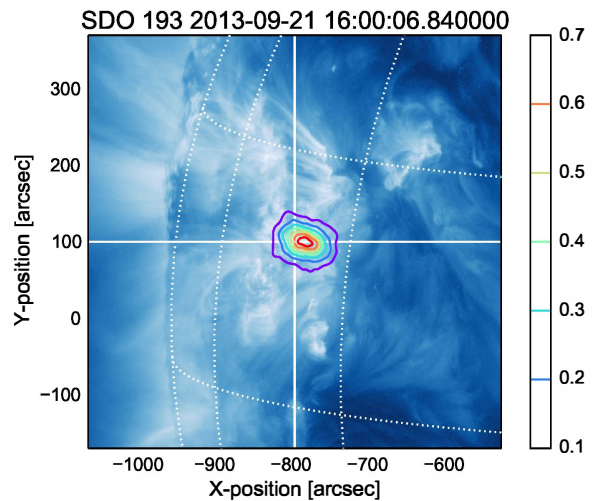

Fig. 5: The HEROES solar pointing in solar coordinates. For context a 193 angstrom EUV image from SDO/AIA of active region \#11850, the HEROES solar science target, is shown in the background. The field of view of this image is representative of that provided by the HEROES x-ray optics. The contours show the probability density of the pointing vector during the HEROES solar observations. The vertical and horizontal lines show the desired pointing location.

$25 \operatorname{arcsec}$ of the target $50 \%$ of the time. The pointing jitter in helioprojective coordinates [10] can be seen in Figure 5 with a reference image from the AIA telescope on the Solar Dynamics Observatory [11] in the background. In this coordinate system, HEROES achieved a pointing jitter standard deviation of $\sim 28$ arcsec from the desired solar target, active region \#11850. A small constant offset of 11 arcsec was found between the desired target and the actual target and is currently under investigation.

\section{The Roll AsPect System (RAS)}

The HEROES payload undergoes a variety of motions in the roll direction, but since the HEROES pointing control system does not control the roll of the gondola, no real-time knowledge of roll aspect is necessary. The roll aspect for astrophysics observations is determined using the star camera, but the star camera cannot be used during solar observations due to the sensitivity of the star camera. The SAS instead includes an independent Roll Aspect System (RAS) for solar observations.

The RAS measures payload roll using the horizon as an external reference. A silvered, knife-edge, right-angle prism combines opposing views of the Earth horizon into a single image (Figure 6), as recorded by an Imperx Bobcat IGVB1310 CCD camera. At float altitude, the horizons are about 6 degrees below the horizontal, with black space above the horizons, so overlapping the opposing horizon views does not compromise either view. As the payload rolls, the two horizons will move in a coupled fashion in the combined image. A red filter (600 nm cutoff) reduces atmospheric haze, and the $12 \mathrm{~mm}$ f/1.8 lens provides a FOV of 23 degrees by 17 degrees with a plate scale of $1.06 \mathrm{arcmin} / \mathrm{pixel}$. The RAS assembly is mounted to the gondola (see Figure 1) to obtain unobstructed horizon views (Figure 7).

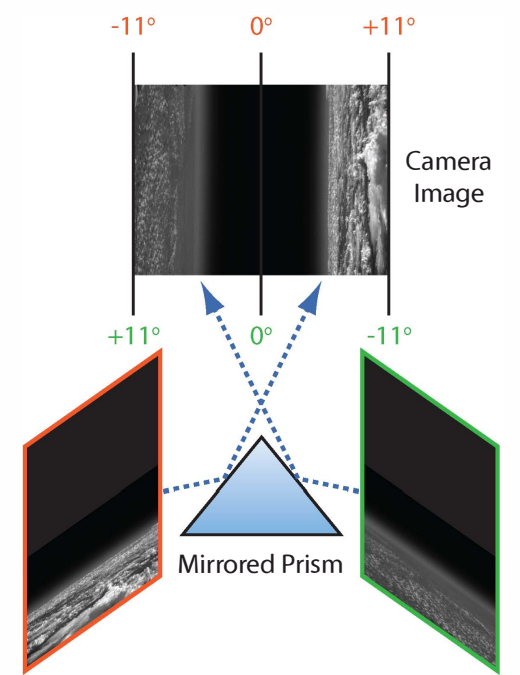

Fig. 6: An illustration of the RAS concept using images from flight. A mirrored prism combines the horizon views from opposite directions into a single camera image. At float altitude, the horizons are about 6 degrees below the horizontal and therefore do not obscure each other. As the payload rolls, the two horizons will shift in tandem to the left or to the right.

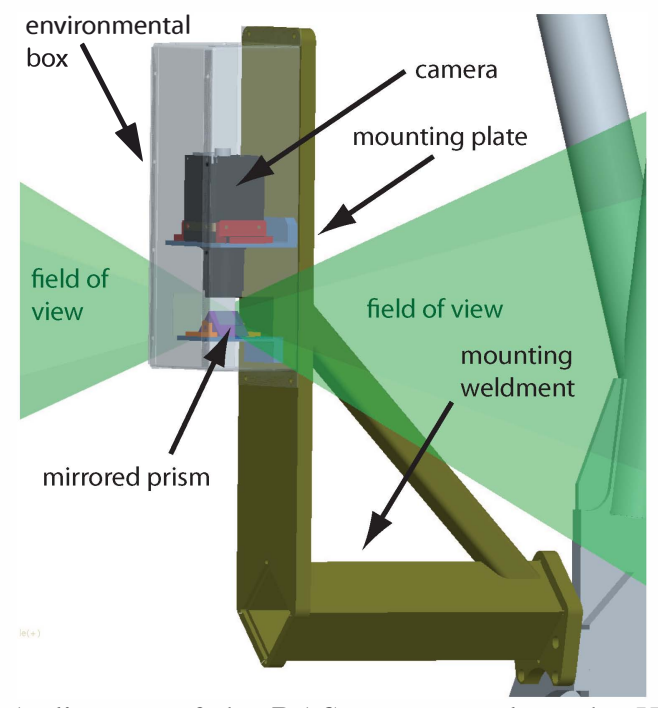

Fig. 7: A diagram of the RAS as mounted on the HEROES gondola. The camera and prism are protected by an aluminum box. Rectangular cutouts on the sides provide unobstructed fields of view (shown in green). The camera and prism are mounted on brackets held by a metal plate. The mounting plate is attached to the gondola through a mounting weldment which is bolted onto the gondola. This position was chosen so as to be as closely coupled to the optical bench as possible to minimize roll offsets introduced by flexing of the gondola structure.

The location of the opposing horizons provides absolute-roll knowledge, and the coupled motion of the opposing horizons provides relative-roll knowledge. RAS images are stored at $4 \mathrm{~Hz}$ to onboard solid-state storage, with the roll aspect determined through post-flight analysis. 

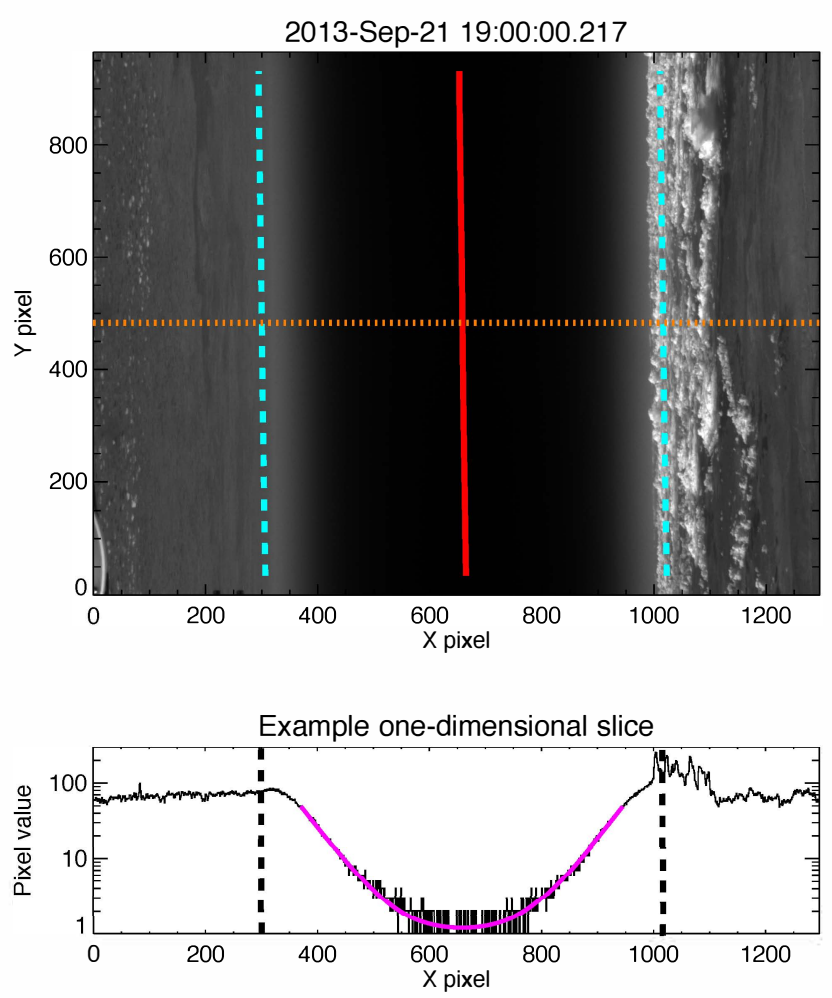

Fig. 8: (Top) An example image from the RAS. The solid, red vertical line marks where the midline between the two horizons as determined from fitting the atmospheric haze. The dashed, cyan vertical lines mark the approximate location of the horizon of the Earth's surface. The dotted, orange horizontal line marks the location of the $1 \mathrm{D}$ cut shown in the following panel. (Bottom) A 1D cut of the above 2D image. The magenta line shows the fit to the exponential fall-off of the atmospheric haze.

\section{A. Flight Performance}

The top panel of Figure 8 shows an image recorded during the actual flight. Local weather conditions on 2013 Sep 21 consisted of significant cloud cover to the west and clearer skies to the east. The horizon itself is difficult to identify precisely, especially when obscured by clouds, and thus is not directly analyzed. Instead, the exponential fall-off of the atmospheric haze above the horizon is used in the analysis to determine relative and absolute roll. This haze was found to be insensitive to the underlying cloud cover.

The bottom panel of Figure 8 shows a one-dimensional cut of the same image, with an illustration of the fit to the exponential fall-off of the atmospheric haze. The model used for fitting consists of two exponential functions with the same scale height (equivalent to a hyperbolic cosine function) plus a constant offset. Thus, the position and motion of the center of the fitted model provides the roll knowledge.

The RAS recorded images throughout the solar observation, with only minor data gaps. Prior to 19:00 UT on 2013 Sep 21, the RAS images are saturated due to improper gain settings for the amplifiers in the camera. Although the horizon itself is saturated, the exponential fall-off of the atmospheric haze is unaffected at higher altitudes and thus roll can still be determined. Following a complete power cycle of the RAS and modifications to the camera's operating parameters, the RAS images are of good quality after 19:00 UT and for the majority of the solar observations.

The payload roll over the $\sim 9$ hours of RAS measurements on 2013 Sep 21 reveal a number of components of roll motion with different frequencies and amplitudes (Figure 9, top). Variations at low frequencies with a timescale of hours and a peak-to-peak amplitude of $\sim 10$ arcmin are likely due to the shifting balance of the gondola as it tracks the Sun. Variations at middle frequencies with a timescale of $\sim 5$ minutes are likely from perturbations produced by the pointing control system and other transient perturbations (e.g., wind). Variations at high frequencies with a very stable period of 24 seconds are the pendulum motion of the gondola-balloon system (Figure 9, middle). Finally, Fourier analysis reveals even higher frequency components with periods of $\sim 1.5$, $\sim 2.3$, and $\sim 8.4$ seconds, which are likely vibrational modes of the gondola and/or cable ladder. Note that the HEROES payload experienced relatively calm winds, and other payloads may experience stronger perturbations or a larger-amplitude pendulation.

The relative roll accuracy from the post-flight analysis is better than $\sim 0.3$ arcmin, which corresponds to, at worst, a $\sim 0.1$ arcsec smear for sources at the solar limb.

\section{B. Comparison to the Star Camera}

Unlike the star camera, which can determine roll aspect for only non-solar observations, the RAS can provide roll knowledge for both solar and astrophysics observations, as long as the Sun is sufficiently high in elevation to evenly illuminate both horizon views. This overlap in roll-aspect capability allows for a comparison, and ultimately a crosscalibration, between the RAS and the star camera.

The first astrophysics target directly following the solar observation was GRS1915+105, and both the star camera and the RAS measured roll during the time period 23:00-23:50 UT (Figure 9, bottom). There is good agreement between the two measurements of payload roll, except near some of the peaks. The entire data set of star-camera solutions is plotted; identifying and filtering out solutions with large uncertainties may resolve some of this disagreement. Note that the star camera, with its slower cadence of $\sim 7.2 \mathrm{~s}$, is unable to clearly resolve the 24 -second pendulation motion.

\section{CONCLUSION}

The HEROES flight has demonstrated the capabilities of a new solar aspect system. Developed in only a year, the SAS has provided stable and accurate solar pointing knowledge for the HEROES payload to make new solar HXR observations. Combined with the existing HERO pointing control system, the PYAS has provided pointing stability to within 25 arcsec of the target $50 \%$ of the time. In addition, the SAS has provided solar pointing knowledge at $3.9 \mathrm{~Hz}$ to better than 10 arcsec, satisfying all of the pointing requirements of the HEROES mission (see Table I). HEROES has provided an important test 

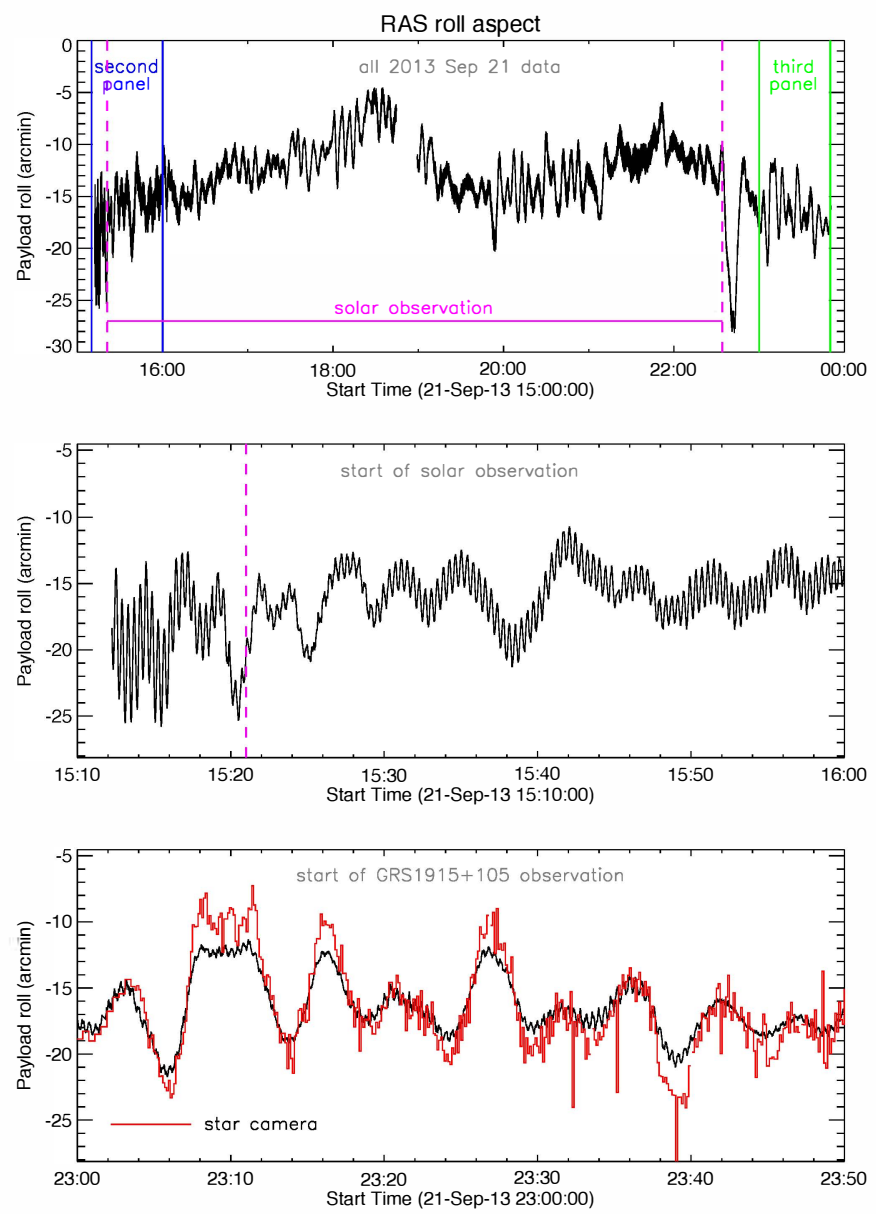

Fig. 9: (Top) The derived payload roll for $\sim 9$ hours on 2013 Sep 21. Note the low-frequency variation with a timescale of hours and the mid-frequency variation with a timescale of $\sim 5$ minutes. The dashed, magenta vertical lines mark the period of solar observation. The solid, blue vertical lines mark the time period for the second panel. The solid, green vertical lines mark the time period of the third panel. (Middle) The derived payload roll at the start of solar observation. Note the high-frequency pendulation with a period of 24 seconds on top of mid-frequency variations. The dashed, magenta vertical line marks when the pointing control system began tracking the Sun. (Bottom) The derived payload roll at the start of the GRS1915+105 observation, with the (preliminary) payload roll derived by the star camera (red) shown in comparison. There is good agreement between the measurements by the two systems.

bed for the upcoming launch of the GRIPS balloon payload, which will use the RAS as well as a modified version of the PYAS. The development of the SAS will also benefit SuperHERO, a proposed balloon payload with improved optics as well as new solid-state pixelated HXR detectors which will investigate both HXR emission from solar flares and astrophysical sources.

\section{ACKNOWLEDGMENT}

The authors acknowledges the Hands-On Project Experience (HOPE) Program for their support of this effort. HOPE is an award presented annually by the NASA Academy of Program/Project \& Engineering Leadership (APPEL), in partnership with NASA's Science Mission Directorate (SMD), Office of the Chief Engineer (OCE) and Office of the Chief Technologist (OCT). The HOPE program manager is David Pierce. We also thank the HERO project for lending their payload and experience and to thank both Marshall Space Flight Center and Goddard Space Flight Center for their support. This research made use of SunPy, a communitydeveloped Python package for solar data analysis[12].

\section{REFERENCES}

[1] J. Gaskin, J. Apple, K. S. Chavis, K. Dietz, M. Holt, H. Koehler, T. Lis, B. O'Connor, M. R. Otero, J. Pryor, B. Ramsey, M. RinehartDawson, L. Smith, A. Sobey, C. Wilson-Hodge, S. Christe, A. Cramer, M. Edgerton, M. Rodriguez, A. Shih, D. Gregory, J. Jasper, and S. Bohon, "High Energy Replicated Optics to Explore the Sun: Hard X-ray balloon-borne telescope," in Aerospace Conference, 2013 IEEE, 2013, pp. 1-11.

[2] S. Christe, A. Shih, M. Rodriguez, A. Cramer, K. Gregory, M. Edgerton, J. Gaskin, C. Wilson-Hodge, J. Apple, K. Stevenson Chavis, A. Jackson, L. Smith, K. Dietz, B. O'Connor, A. Sobey, H. Koehler, and B. Ramsey, "The high energy replicated optics to explore the sun mission: a hard x-ray balloon-borne telescope," in SPIE Optical Engineering + Applications. SPIE, Sep. 2013, pp. 886 206-886206-13.

[3] B. D. Ramsey, C. D. Alexander, J. A. Apple, C. M. Benson, K. L. Dietz, R. F. Elsner, D. E. Engelhaupt, K. K. Ghosh, J. J. Kolodziejczak, S. L. O'Dell, C. O. Speegle, D. A. Swartz, and M. C. Weisskopf, "First Images from HERO, a Hard XRay Focusing Telescope," The Astrophysical Journal, vol. 568, no. 1, pp. 432-435, Mar. 2002.

[4] A. Y. Shih, R. P. Lin, G. J. Hurford, N. A. Duncan, P. Saint-Hilaire, H. M. Bain, S. E. Boggs, A. C. Zoglauer, D. M. Smith, H. Tajima, M. S. Amman, and T. Takahashi, "The Gamma-Ray Imager/Polarimeter for Solar flares (GRIPS)," in SPIE Astronomical Telescopes + Instrumentation. SPIE, Sep. 2012, pp. 84434H-84434H-15.

[5] B. F. O'Connor, "Thermal Control of the Balloon-Borne Telescope HEROES," in 43rd International Conference on Environmental Systems. Reston, Virginia: American Institute of Aeronautics and Astronautics, Jul. 2013.

[6] M. Fivian, R. Hemmeck, A. McHedlishvili, and A. Zehnder, "RHESSI Aspect Reconstruction,” Solar Physics, vol. 210, no. 1/2, pp. 87-99, 2002.

[7] H. Moon, R. Chellappa, and A. Rosenfeld, "Optimal edge-based shape detection," Image Processing, IEEE Transactions, vol. 11, no. 11, pp. 1209-1227, Nov. 2002.

[8] E. Parzen, "On Estimation of a Probability Density Function and Mode," The Annals of Mathematical Statistics, vol. 33, no. 3, pp. 1065-1076, Sep. 1962

[9] M. Rosenblatt, "JSTOR: The Annals of Mathematical Statistics, Vol. 27, No. 3 (Sep., 1956), pp. 832-837," The Annals of Mathematical Statistics, 1956.

[10] W. T. Thompson, "Coordinate systems for solar image data," Astronomy and Astrophysics, vol. 449, no. 2, pp. 791-803, Apr. 2006.

[11] J. R. Lemen, A. M. Title, D. J. Akin, P. F. Boerner, C. Chou, J. F. Drake, D. W. Duncan, C. G. Edwards, F. M. Friedlaender, G. F. Heyman, N. E. Hurlburt, N. L. Katz, G. D. Kushner, M. Levay, R. W. Lindgren, D. P. Mathur, E. L. McFeaters, S. Mitchell, R. A. Rehse, C. J. Schrijver, L. A. Springer, R. A. Stern, T. D. Tarbell, J.-P. Wuelser, C. J. Wolfson, C. Yanari, J. A. Bookbinder, P. N. Cheimets, D. Caldwell, E. E. DeLuca, R. Gates, L. Golub, S. Park, W. A. Podgorski, R. I. Bush, P. H Scherrer, M. A. Gummin, P. Smith, G. Auker, P. Jerram, P. Pool, R. Soufli, D. L. Windt, S. Beardsley, M. Clapp, J. Lang, and N. Waltham, "The Atmospheric Imaging Assembly (AIA) on the Solar Dynamics Observatory (SDO)," Solar Physics, vol. 275, no. 1-2, pp. 17-40, Jun. 2011.

[12] S. Christe, A. Y. Shih, J. Ireland, D. Perez-Suarez, S. Mumford, V. K. Hughitt, R. Hewett, F. Mayer, and S. D. Team, "A Community Python Library for Solar Physics (SunPy)," American Astronomical Society, vol. 44, Jul. 2013 


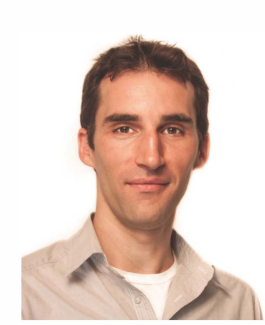

Steven Christe Dr. Steven Christe is a Research Astrophysicist in the Solar Physics Laboratory, part of the Heliophysics division at the NASA Goddard Space Flight Center in Greenbelt Maryland. His research interests focus on the study of particle acceleration in solar energetic events through the study of hard X-ray emission from solar flares and the quiet Sun with particular emphasis on the statistics of small transient bursts such as solar microflares; hard X-ray emission associated with solar radio emission; and the application of hard X-ray focusing optics to solar observations. Dr. Christe completed his Ph.D. in 2007, under the supervision of Prof. R.P. Lin and Dr. S. Krucker. During his graduate studies, Dr. Christe developed a sounding rocket program to study particle acceleration in solar microflares by applying newly-developed hard X-focusing optics combined with pixelated solid-state detectors to solar observations. The FOXSI program, short for the Focusing Optics X-ray Solar Imager, is an ongoing partnership between the Space Sciences Lab UCB, MSFC, and the Japanese Astro-H mission. As a postdoctoral researcher at the Space Sciences Lab, Dr. S. Christe oversaw the FOXSI program as the project manager/project scientist and now as Co-PI. Dr. Christe became a civil servant at NASA Goddard Space Flight Center in the Fall of 2009. He was a member of the team that developed the RAM, SEE2020, and FOXSI mission concepts for the Heliophysics decadal survey. He has been working with the GSFC microcalorimeter group to apply new transition-edge microcalorimeters to solar observations since 2010. He led the successful launch of FOXSI in 2012 and is the heliophysics PI for the High Energy Replicated Optics to Explore the Sun mission (HEROES), a partnership between GSFC and MSFC, to modify and fly an existing astrophysics balloon payload to observe the Sun in hard X-rays $(>20 \mathrm{keV})$.

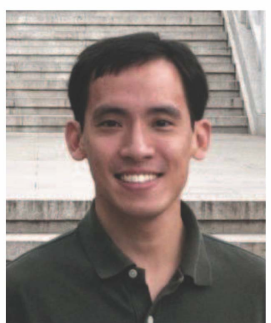

Albert Y. Shih joined the Solar Physics Laboratory at GSFC in 2010. His research interests focus on $\mathrm{X}$-ray and gamma-ray observations of particle acceleration in solar flares. He is currently serving as the Deputy Mission Scientist for RHESSI, and is the project manager and project scientist for Gamma Ray Imager/Polarimeter for Solar Flares (GRIPS). Dr. Shih is the solar project scientist for HEROES.

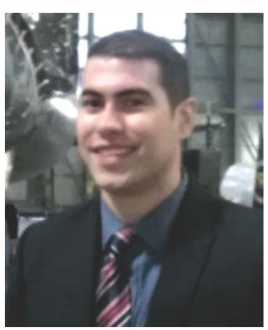

Marcello Rodriguez Started at GSFC full time in 2007. He has worked on the Lunar Reconnaissance Orbiter (LRO) spacecraft purification system and has since worked on instrumentation development that includes UV imagers, ion/neutral mass spectrometers in the Heliophysics Division. He has a M.S. in Applied Physics from John Hopkins University. Mr. Rodriguez is the SAS Systems Engineer for HEROES.

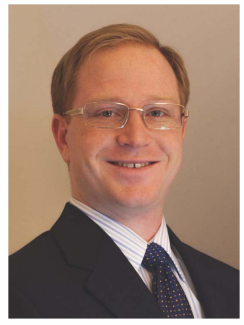

Kyle Gregory studied electrical engineering at the University of Pennsylvania as well as the University of Maryland College Park. He joined GSFC in 2005, worked on a number of projects including the Gravitational and Extreme Magnetism Small Explorer (GEMS), where he developed FPGA firmware for the main science instrument. He also worked on the Soil Analysis at Mars (SAM) mission, where he designed and conducted integration validation testing. He is the SAS Electrical Engineer.

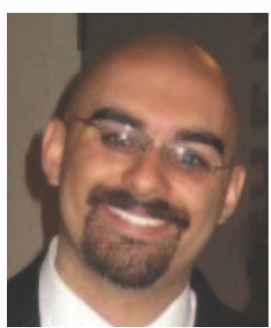

Alexander Cramer studied mathematics and electrical engineering as an undergraduate at the University of Maryland, receiving BS degrees in both in 2009. $\mathrm{He}$ has worked as an Electrical Engineer at GSFC since 2009 . He is currently working toward a $\mathrm{PhD}$ in machine vision at the University of Maryland. Mr. Cramer is the SAS Software Engineer.

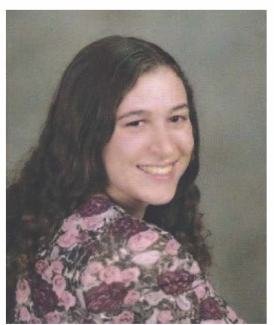

Melissa Edgerton is an aerospace engineer for NASA at GSFC. She earned a B.S. in Mechanical Engineering and is currently pursuing an M.S. in Aerospace Engineering from the University of Maryland in College Park. Melissa spent two years as a co-op student at Goddard before converting to a full-time employee three years ago. She is working in the Electromechanical Systems Branch with a focus on Optomechanics. Ms. Edgerton is the SAS Mechanical Engineer.

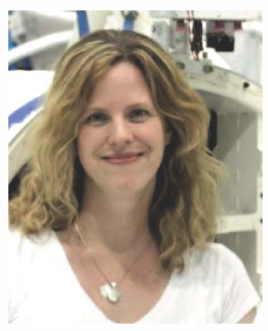

Jessica Gaskin is a physicist in the X-Ray Astronomy Group at NASA Marshall Space Flight Center in Huntsville, AL. She has characterized multiple types of solid state detector systems for X- Ray Astronomy and has supported the High Energy replicated Optics (HERO) hard X-ray balloonborne telescope; participating in flight campaigns and detector calibration. Over the past few years, she has focused her research on Planetary/In- Situ based instrumentation, concentrating on the miniSEM. She has a B.S. in Physics (specializing in Astrophysics) from New Mexico Tech, an M.S. in Astronomy from Case Western Reserve University, and a Ph.D. in Physics from the University of Alabama in Huntsville. Dr. Gaskin is the MSFC PI for HEROES.

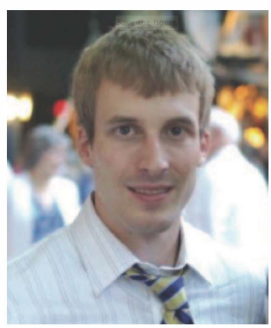

Brian O'Connor joined MSFC as a Co-Op in 2006. He is a Thermal Engineer in the Thermal \& Mechanical Analysis Branch of the Space Systems Department. He has worked on a number of projects supporting the thermal subsystem team. For the ILN, he performed an extensive analytical trade of radiator designs. Mr. O'Connor is the Thermal Engineer for HEROES.

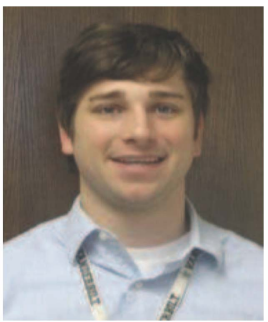

Alexander Sobey joined MSFCs Federal Career Intern Program as an Aerospace Engineer. Since then, he has taken on a structural design role for the Pre-Phase-A study of the Energetic X-ray Imaging Survey Telescope (EXIST). Mr. Sobey has also worked on the International Lunar Network (ILN) as well as the Extreme Universe Space Observatory (EUSO) as the structural designer. Mr. Sobey is the lead Mechanical Engineer for HEROES 